Research Article

\title{
Low-Cost, Low-Energy, Wireless Hydrological Monitoring Platform: Design, Deployment, and Evaluation
}

\author{
Qasem Abdelal $\mathbb{D D}^{1}$ and Ahmad Al-Hmoud $\mathbb{D D}^{2}$ \\ ${ }^{1}$ Department of Civil and Environmental Engineering, German Jordanian University, Amman 11180, Jordan \\ ${ }^{2}$ Department of Energy Engineering, German Jordanian University, Amman 11180, Jordan \\ Correspondence should be addressed to Ahmad Al-Hmoud; ahmad.alhmoud@gju.edu.jo
}

Received 1 July 2020; Revised 12 January 2021; Accepted 20 January 2021; Published 10 February 2021

Academic Editor: Xavier Vilanova

Copyright ( 2021 Qasem Abdelal and Ahmad Al-Hmoud. This is an open access article distributed under the Creative Commons Attribution License, which permits unrestricted use, distribution, and reproduction in any medium, provided the original work is properly cited.

\begin{abstract}
Climate change has increasingly been considered responsible for irregular weather patterns leading to many environmental hazards and catastrophes. Coping with these conditions and providing effective solutions require monitoring and collecting data of various hydrological parameters and events in high spatial and temporal resolutions, which is generally limited by the cost and energy requirements of the monitoring devices. In this work, we push the limit of the current low-cost data acquisition capabilities by developing the HydroMon3: a hydrological monitoring platform that collects, stores, and transmits high temporal resolution data reliably and accurately, and capable of interfacing different types of sensors. The modular design is driven by utilizing the recent burst in commercially available IoT-related electronics modules to minimize the cost and maximize flexibility, while applying various hardware and software techniques to ensure reliability and energy performance. Stream stage and tipping bucket monitoring units based on the HydroMon3 platform were deployed to more than 20 locations in two different watersheds, and their performance over a 6-month season was evaluated. Collected data for a number of storms provided important insights for linking hydrological events and showed substantial variability in the monitored parameters both spatially and temporally, which were compared with local data records and confirmed that conventional hydrological data acquisition methods are under representative of the actual events. Field-proven results demonstrate the units' ability to maintain autonomous operation from several months for the stream stage monitors to years for the rainfall gauges using of-the-shelf AA batteries.
\end{abstract}

\section{Introduction}

One of the established impacts of climate change is irregular, more intense precipitation patterns and ambient temperature fluctuations. This has a significant impact on the occurrence of flash floods and droughts [1]. Apart from the catastrophic loss in lives, floods account for extreme economic losses, and it has been reported that in Europe alone, the cost of damage was more than $\$ 500$ billion between 1980 and 2015 [2]. In semiarid regions, Jordan as an example, flash floods pose a very serious threat to lives and property $[3,4]$, several unfortunate events in which lives were lost were witnessed in Petra and near the Dead Sea.

Optimal water monitoring and management are necessary. Hydrological monitoring is critical for water resources management and flood risk mitigation studies, it can be achieved through a series of sensors and data loggers, and these can be used for several applications, examples of which are precipitation monitoring systems, solar radiation [5], soil moisture monitoring [6], flood monitoring [1, 7, 8], and monitoring of aquatic environments [9]. Input from all these sensor systems will help better analyze existing conditions and make better decisions. Classical data loggers and SCADA systems can be very expensive and provide limited options for customization and integration with other systems. Data is either stored locally or transmitted through cables, which adds high installation costs and limits the possible commissioning area [1]. Some wireless solutions exist, and they are very expensive and provides even less system flexibility. Complete, widespread sensor networks can be cost prohibitive, thus 
arising the need for developing low-cost, low-energy, autonomous wireless systems.

In the past decade, major interest was put in the development of wireless sensor networks and the Internet of Things (IoT) [10]; in the water resources field, they are envisioned to transform water resources management by allowing for realtime monitoring and control [11]. Wireless sensor networks (WSN) provide a very promising solution for environmental monitoring applications, where many spatially scattered measurement points of interest exist, and long-term data acquisition is needed.

Several studies addressed hydrological monitoring and flood warning systems. Abdullahi et al. [8] designed a flood monitoring system that integrates both flow and water level sensors and uses two class neural networks to predict flood status. The system was laboratory based and used an ESP8266-based NodeMCU board to transmit data over WiFi to a cloud server. Nuhu et al. [12] designed a laboratorybased water level measurement system and focused on enhancing the energy efficiency of data transmission using the XM1000 module-which is a radio frequency module based on the Texas Instruments CC2420 chip-by utilizing the Internet Protocol Version 6 over Low Power Wireless Personal Area Network (6loWPAN) technology. The application compares current water level with a predetermined threat level and alerts relevant entities via email when a flood is eminent. The work of Nuhu et al. [12] is based on an emulation in which the results look promising.

Kruger et al. [13] developed a system for river stage monitoring designed to be bridge mounted, each unit is equipped with an ultrasonic sensor, a GPS receiver, and a solar panel for power supply. Data is transmitted through cellular networks. Stage data is frequently measured and transmitted to be available over the internet. Bartos et al. [11] developed an end-to-end system demonstrating an autonomous water system, with a focus on urban storm water management. The authors comment that technology is no longer a limitation for achieving autonomous water systems; rather, it is the proprietary nature of the equipment, thus their high cost, which is limiting their widespread usability; in addition, there is a lack of proven case studies where these smart water management systems can be used and, lastly, the lack of the end-to-end solution capable of supporting water resource management applications. To that end, they developed their system of open source software, hardware, and cloud computing services; the developed systems were tested in two locations: one addressing flash flood problems, whereby a set of sensors were deployed to measure stream stages, in addition to a wide array of other meteorological and soil moisture measurements. The other location was for monitoring and management of a small watershed storm water system, in which valves and gates were automatically controlled by the system, thereby optimizing water usage, enhancing residence time within detention basins, and improving overall water quality.

Perez et al. [1] summarized some of the criteria that need to be achieved these sensor systems; for example, power usage needs to be optimized for deployment in remote locations, they also need to be reliable and be able to withstand harsh weather conditions, and in addition, the systems must be able to accommodate several sensor types and allow for long-range communication. Flood monitoring has been done using various techniques, ranging from image processing utilizing the widely used surveillance cameras $[14,15]$, to the use of ultrasonic sensors [13], to the deployment of mobile sensors that submit GPS location signal to measure flow velocity [16].

Previous work has demonstrated promising results and developed a number of successful prototypes. However, the number of field-operational units is still limited, with only a few cases demonstrated in literature. Moreover, cost and energy efficiency have room to be improved and optimized, which is essential to maximize the benefits of such devices.

In this work, a complete hydrological monitoring platform, named HydroMon3, was designed, built, deployed, and evaluated. Following a modular design concept, the cost and part count of the platform were significantly reduced and greatly increased the flexibility of the design. Several hardware and software techniques were utilized to push the envelope on the energy efficiency, achieving an autonomous operation of several months to years using of-the-shelf AA batteries. Software-based probabilistic filtering algorithms were optimized to achieve accurate results with minimal energy consumption. More than 20 devices were deployed in two hydrological catchments in Jordan, one is the Zarqa Main catchment discharging to the Dead Sea with an approximate area of $270 \mathrm{~km}^{2}$, and the other is in Petra with an approximate area of $80 \mathrm{~km}^{2}$. Both locations are prone to occasional thunderstorms and consequently flash floods. All streams in the two watersheds are ephemeral, and thus, water will flow only in case of rain. The deployed units mainly monitor precipitation rate and stream stages, while providing other data like ambient temperature and humidity. The data is logged locally and transmitted over the GSM network to the MathWorks ${ }^{\circledR}$ Thingspeak $^{\mathrm{TM}}$ cloud service where it can be stored, viewed, and analyzed.

In the following sections, the design and challenges in achieving good accuracy, reliability, and energy efficiency will be discussed, followed by evaluation of the field performance and demonstration of the end results.

\section{Materials and Methods}

2.1. Main Platform Hardware Description. The concept of the platform presented in this work relies on taking advantage of the wide range of readily available electronics and modules to build a low-cost, flexible, and easy-to-reproduce platform, enabling us to acquire high-resolution hydrological data both temporally and spatially.

The HydroMon3 is a low-cost, low-energy, wireless platform, designed using a modular design concept to accommodate different hydrological sensors for maximum flexibility. The device serves as a platform to receive data from the sensors, process it, store it, and transmit it. The main platform consists of a microcontroller, a real-time clock (RTC), a local storage unit, and a GSM module. Depending on the objective of the system, multiple sensor combinations could be connected to the main platform. A block diagram of the main platform components is shown in Figure 1. 


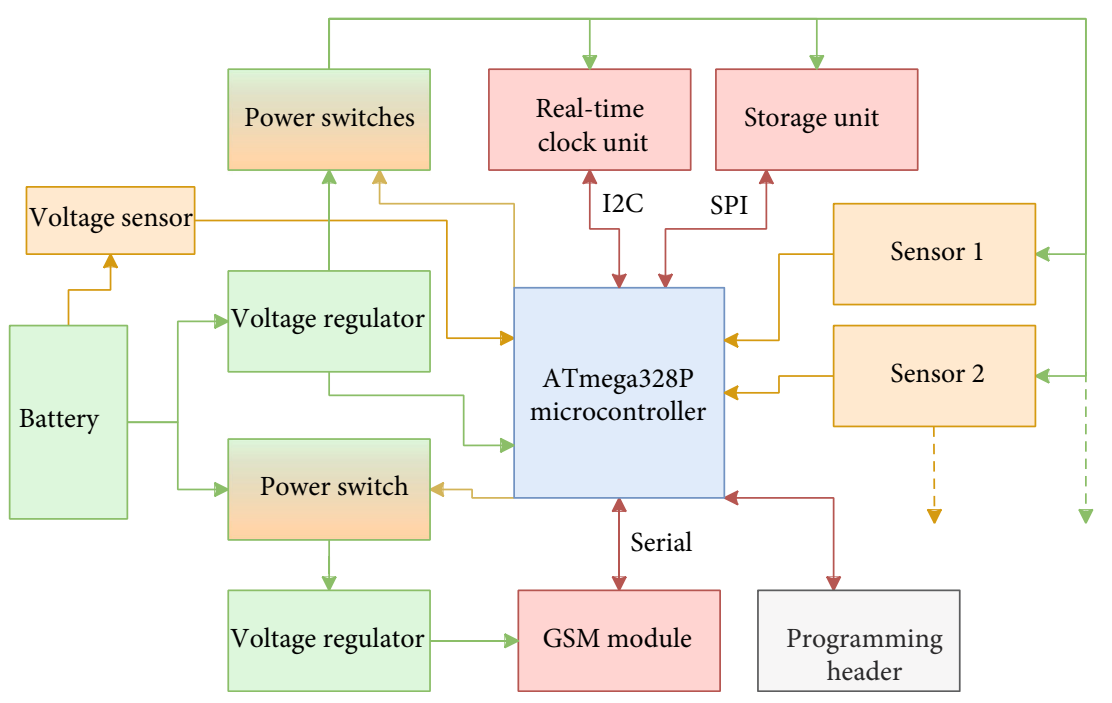

Figure 1: Block diagram of the major HydroMon3 components.

The main platform was designed following a modular design concept to maximize flexibility and prioritized the use of commercially available modules to lower cost, increase availability, and speed up production. Naturally, in order to achieve very high energy efficiency, some hardware modifications were applied to the modules, and the power management circuit was custom designed.

The microcontroller unit (MCU) is the functional brain of the platform, managing and processing data, controlling other modules, and making decisions. An Atmega328P mounted on an "Arduino Pro Mini" board is used because of its suitable performance and memory, several communication types available (Serial, SPI, I2C), wide voltage operating range, low-energy capabilities, and low cost. While the Atmega328P features a number of timers, the use of a dedicated RTC is more accurate and provides better code efficiency within the MCU. Furthermore, the Atmega328P timers turn off during some levels of deep sleep to save energy, and keeping them on all the time uses substantially more energy than the dedicated RTC. Therefore, the performance of the MCU and the overall energy efficiency are better using the dedicated RTC. A ZS-042 module based on the DS3231 RTC provided good accuracy, internal temperature compensation, and low cost. The RTC module is connected to the MCU through the I2C connection port.

A SIM8001 GSM module was used to transmit data using GPRS over a $2 \mathrm{G}$ network. Compared to higher bandwidth options like WiFi, 3G, and $4 \mathrm{G}$ networks, 2G GSM networks have the widest coverage area, which is particularly important in the remote areas where the device is deployed; in addition, considering the small size of data packs transmitted, the relatively low bandwidth of the $2 \mathrm{G}$ network will not be a concern. The GSM module is controlled by the MCU using AT commands through a serial connection port. Machine to Machine (M2M) SIM cards provide low-cost access to the network and are suitable for mass deployment of the devices.

In addition to transmitting the data, a local storage is used as a backup in case of any issues in the transmission process. A microSD card storage module was used as the storage media allows for easy data extraction and replacement. The module used is based on the SN74LVC125A quadruple bus buffer gate IC, which is connected the MCU via the SPI connection port.

The HymdroMon3 platform was fitted with a custom designed power management circuit to achieve three targets, provide suitable voltage level, stable current, and low energy losses. A block diagram of the power management circuit is shown in Figure 2.

A low quiescent current DC/DC converter provides 5 volts to run the MCU, which in turn controls a series of power switches to turn on and off different modules and sensors as needed to minimize energy consumption. The GSM module is powered by a separate secondary power circuit because of the big difference of its power requirements in comparison to the other parts of the circuits. The GSM module requires $4.1 \mathrm{~V}$ and draws short current pulses of up to 3 amperes. A separate DC/DC converter is used to provide the required voltage, and a combination of buffering circuits before and after the converter provides the high-energy pulses required with minimal disturbance to the input voltage, to achieve high stability in the transmission process.

The buffering and noise cancellation circuits are a combination of different types of capacitors and passive components custom designed for each part of the circuit to stabilize the input voltage at different current drawing conditions. The buffering circuits are important under three situations: with short bursts of high current, for sensitive or higher frequency components, and for components relatively far away from the power source. The common source of the possible issues is the parasitic inductance and capacitance in the Printed Circuit Board (PCB) traces. These parasitic components are always present, but their effects are greatly increased under one-or more-of the three situations mentioned above. The use of the buffering circuits was empirically proven to greatly increase the stability and reliability of the overall system. The power switches are a combination of high-side and low-side 


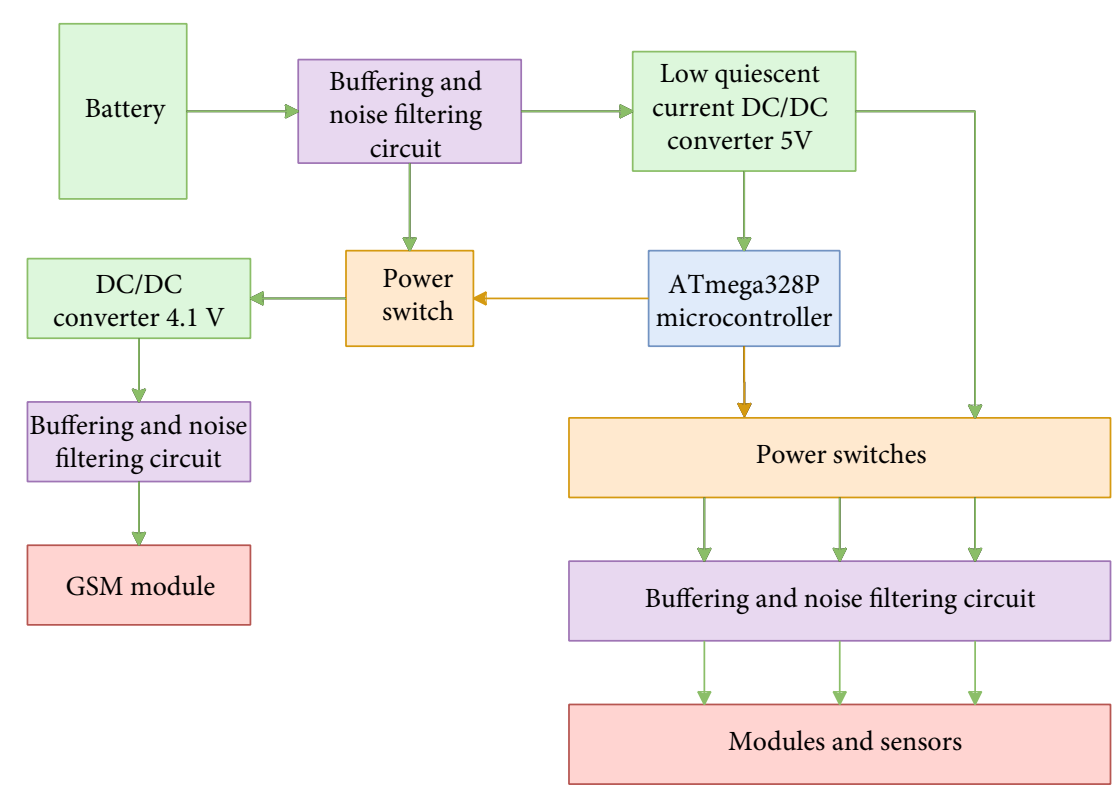

FIGURE 2: Power management circuit block diagram.

MOSFET- or BJT-based switching circuits, depending on the operating nature and switching requirement of the component.

Shown in Figure 3, the PCB layout plays a major role in reducing parasitic components in the traces and reducing electrical interference for the more sensitive parts. Routing parallel power paths utilizing both sides of the PCB minimizes the power loop area, which in turn minimizes the parasitic components in the traces. Using separate ground planes for high power components and more sensitive components reduces electrical interference, and the large ground plane under the MCU provides noise shielding [17]. The populated boards are mounted inside weather-proof boxes equipped with a GSM antenna and a battery pack. A populated board and an assembled unit are shown in Figure 4.

2.2. Energy Considerations. Extended data acquisition periods in remote locations dictates very tight energy usage allowance and imposes various restrictions on the type of power sources. The first step is to ensure minimal energy loss by removing unnecessary hardware. These modifications include removing all LEDs (except for a heartbeat signaling LED) and partial removal of any unutilized parts of the modules or sensors used. The next step is to use physical switches to completely disconnect any part of the circuit when it is not being used. This is very critical, since when dealing with very low current consumption requirements, most sleep modes on chips and modules still allow drawing relatively substantial current. By using a combination of high-side and lowside switching circuits, the leakage current is reduced to that of the transistors making these switching circuits, and those were chosen to have leakage current in the range of tens of nanoamperes or less. The duration the platform components staying on is equally important; therefore, the minimal time each component needs to stay up without compromising reliable performance was empirically determined to optimize energy consumption.
The MCU, which is never disconnected from the power supply, is supplied by a low quiescent current LDO DC/DC converter, which is specifically chosen to limit the standby current consumption of the regulator during the deep sleep mode of the MCU, which is the case most of the time. These overall measures allow for an extremely low average standby current consumption of $30 \mu \mathrm{A}$. This standby current was measured by a Keysight B2901A source/meter unit.

The energy requirements of each component in the system were thoroughly studied to operate it as efficiently as possible, and in order to accomplish that, theoretical average values from datasheets are not sufficient, as most components have many variables that effects the energy consumption behavior. So, an extensive set of tests were undergone to take highresolution current measurement samples from each component, while running it at the extremes of its operating conditions and at the expected conditions of practical operation. This allowed for an accurate representation of the expected real-life energy consumption. The results of our tests are shown in Table 1.

All measurements were done using Keysight B2901A high-precision source/meter unit and were performed under a supply voltage of $7.9 \mathrm{~V}$. The figures of the RTC were not shown here as it is supplied by its own battery, which practically lasts more than 5 years.

As noted by previous research, using common renewable energy sources like solar panels was avoided due to the risk of vandalism and theft [1], in addition to increasing the complexity of field mounting and narrowing the range of locations the devices can be deployed at. In principle, a battery pack with a working voltage range of $5-16 \mathrm{~V}$ can be adequately used to run the device. Yet, the range of $6-10 \mathrm{~V}$ was found to be the most efficient and reliable. Since the device is intended to be deployed in bulk numbers, six common AA alkaline batteries are used in series configuration to achieve the desired operating voltage over the lifetime of the battery and provide 


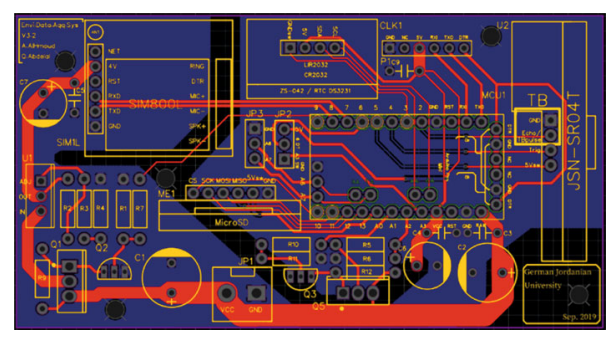

FIgure 3: Main platform PCB layout.

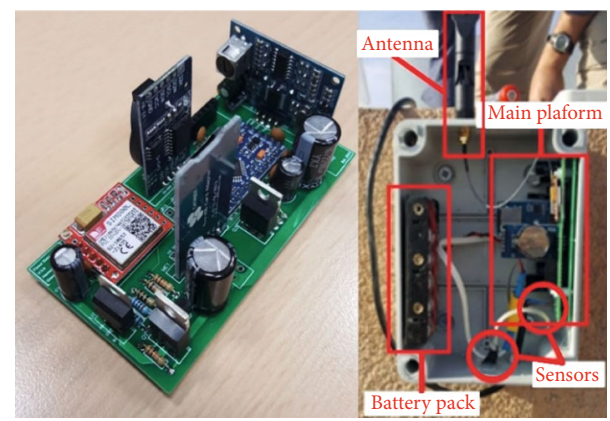

Figure 4: A populated board and a deployed HydroMon3 unit.

adequate energy capacity, while minimizing the overall system size. To increase the lifetime, NiMH, C-cells, D-cells, or lithium batteries can be directly used instead.

2.3. Stream Stage Monitoring. One of the biggest motivations for the development of the HydroMon3 platform is to monitor floods through stream stage measurements and to acquire high-resolution data for characterization and modeling purposes. To achieve that, the device should be able to measure water level with adequate accuracy and temporal resolution.

Due to the mechanical nature of sound waves, ultrasonic sensors are a popular choice to measure water surface distance. In addition to that, several factors make them appealing for our application. This type of sensor provides contactless readings with no moving parts, and no special mounting requirements, which is crucial for applications involving potentially devastating water flow and allows for simpler and more versatile installation. A JSN-SR04T-2.0 waterproof ultrasonic sensor is used for its availability, low cost, and rigidity. The sensor consists of a waterproof transducer connected through a $1.5-\mathrm{m}$ shielded cable to the main board, and it can be easily mounted using commercially available plastic tubing in field deployment. The sensor interfaces to the microcontroller through two digital pins to trigger pulses and receive echo duration.

The flood monitoring system is built upon achieving monitoring goals under very low energy consumption. This dictates an intermittent operation of the MCU, alternating between different sleep and wake-up levels to guarantee adequate level of performance at the least amount of required energy. The general workflow of the platform is shown in Figure 5.
Immediately after the first power-up, the system enters self-testing routine, where it starts applying a number of algorithms to start and diagnose each part of the platform, initiate logging session in the memory, and transmit all diagnostic data to the cloud server to start a transmission session there. This last step doubles as a way to confirm the health status of the transmission subsystem and gives the operator a way to assess the functionality of the device before any extended deployment.

During the main loop, the MCU is in the deepest sleep level most of the time, waking periodically using the internal Watch Dog Timer (WDT) to trigger periodic wake-up cycles, where the code proceeds to acquire main hydrological and meteorological data every prespecified time duration. This interval can be changed depending on the desired data resolution, where the higher data acquisition frequencies impact the overall lifetime of the battery. In Figure 5, two-minute intervals were used, as it was the chosen frequency for the deployed devices. As the transmission process requires a substantially high amount of energy, the data is stored on the MCU's memory temporarily before sending it as a data bulk at the end of a certain time interval. This exact time interval is determined according to the MCU's memory capabilities, data resolution, number of sensors, and how "real-time" the data is desired to be. Due to the use of an RTC, it could be also set to transmit at the end of each hour, as shown Figure 5. This makes data much easier to post process, and simpler to compare with data from other sources.

The RTC could have been used to initiate the trigger to wake the system up, but this would lead the RTC to require much higher energy (around 92 times more as per the datasheet) to operate in this mode, and in case of any failure in the RTC, the entire system would malfunction. On the other hand, our approach requires less energy, and in case of a failure in the RTC, it would send a signal reporting that and use an internal timer instead, which will shorten the battery life substantially but will keep the system running until it a maintenance team can deploy another device.

Due to the very low energy consumption of the device, low-resolution voltage data is more than enough. The voltage readings are taken only once per hour and are taken before the start of the transmission routine, since the transmission's relatively high power demand may cause the battery voltage to slightly dip, making the reading less accurate.

The transmission routine starts by switching on the transmission subsystem power circuit, followed by a series of AT commands through the serial communication ports to guide the GSM module thought the GPRS routine and transmit the bulk data to the cloud. The MathWorks ${ }^{\circledR}$ Thingspeak $^{\mathrm{TM}}$ IoT service is used because of the powerful visualization and postprocessing abilities directly on the website.

To guarantee best accuracy for the water level field measurements, three stages of comprehensive tests were conducted on the system and are as follows.

(i) First Stage. A series of tests were conducted under laboratory-controlled conditions. Measurements were taken at different distances, and over the course of several days, to tweak all power-up, standby, and 
TABLE 1: Current and energy consumption figures for major components.

\begin{tabular}{lccc}
\hline Component & Average current $(\mathrm{mA})$ & Duration of an operation cycle (seconds) & $\begin{array}{c}\text { Energy consumption/cycle } \\
\text { of operation (mWh) }\end{array}$ \\
\hline GSM & 35.16 & 38 & 2.923 \\
Ultrasonic sensor & 20 & 3.3 & 0.145 \\
SD card module & 17.4 & 2.44 & 0.093 \\
MCU + passive components & 0.03 & 3600 & 0.237 \\
\hline
\end{tabular}

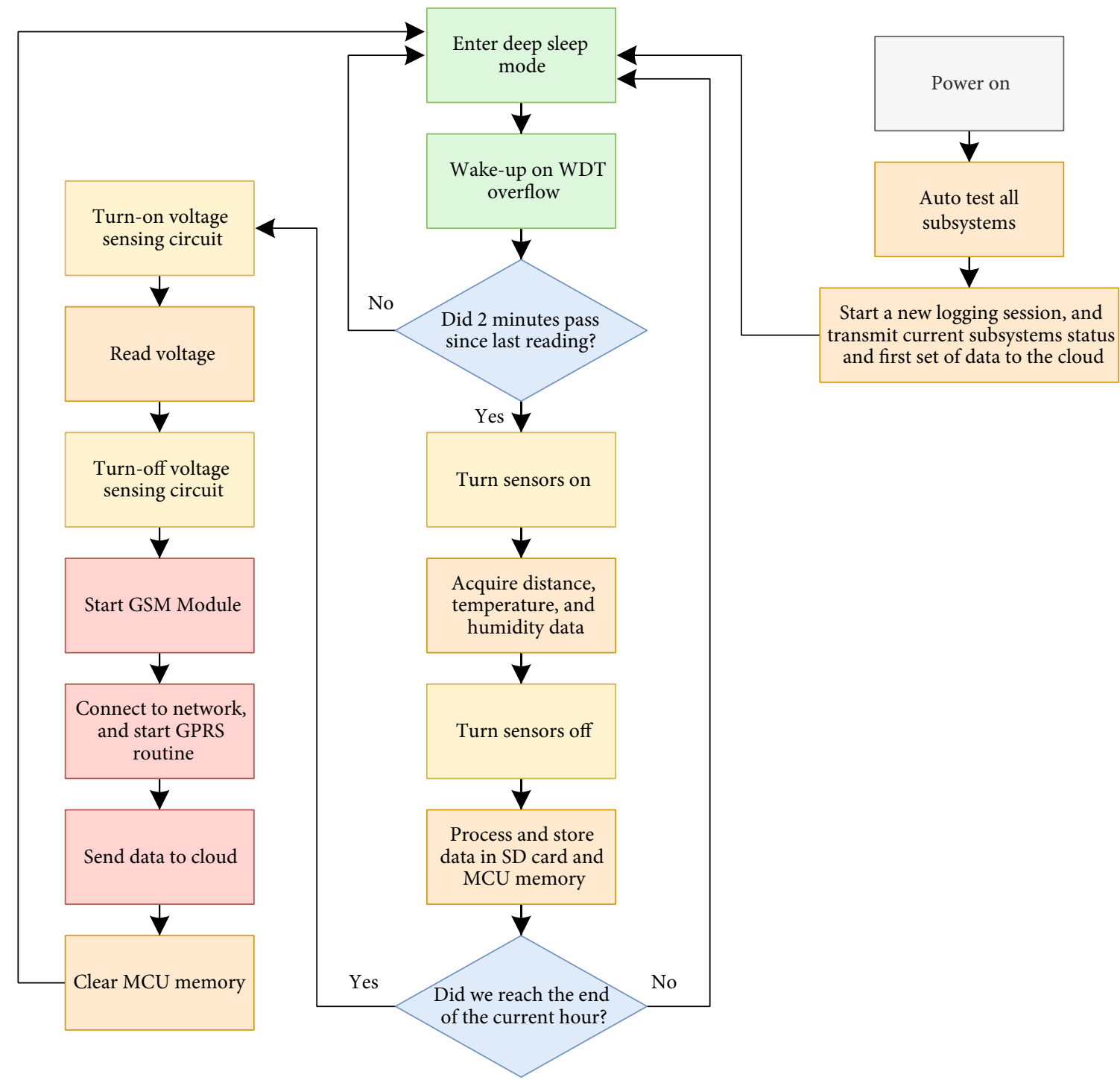

FIGURE 5: The general workflow of the stream stage monitoring system.

pulse timings, in order to minimize energy consumption while guaranteeing readings reliability, repeatability, and voltage stability.

(ii) Second Stage. To simulate uncontrolled conditions expected in the field, the system was tested in an outdoor testing facility, where temperature and humid- ity were changing through the day, and some wind and vibrations were present. The water level was stable, and the measuring angle and surroundings were close to ideal.

The measurements were substantially affected by the ambient temperature and humidity cycles through 


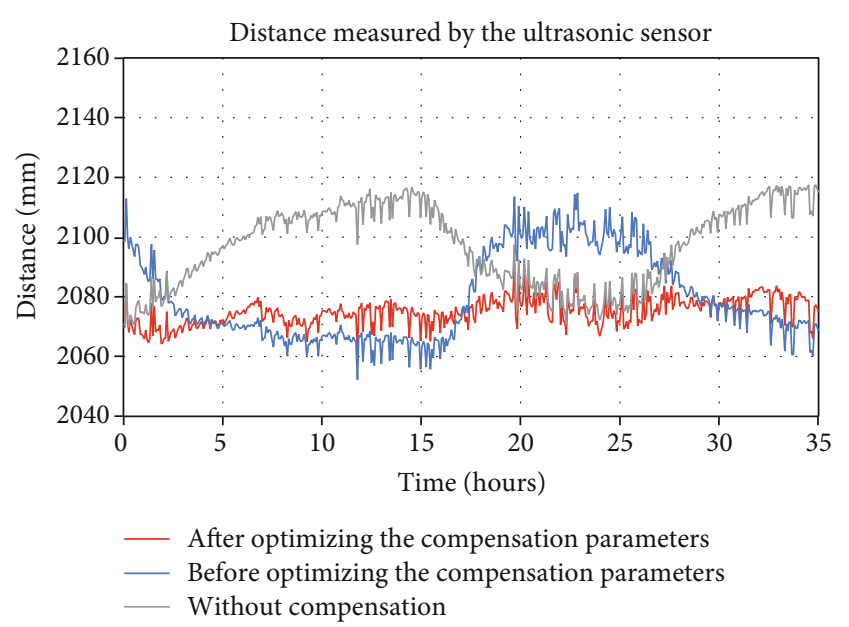

FIGURE 6: Comparison of the distance measurements through the different stages of compensation and optimization.

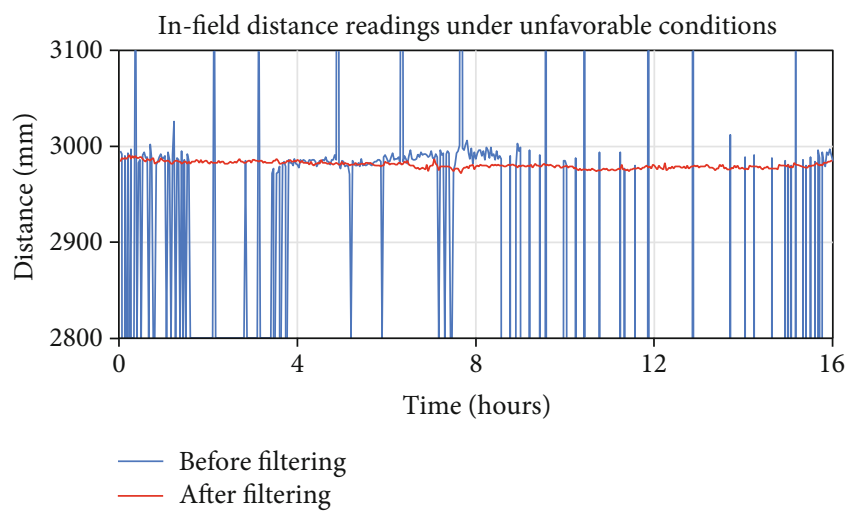

Figure 7: Demonstration of the effectiveness of the developed filtering method.

the day. Taking the measurements without accounting for those variations resulted in substantial variations through the day. To enhance the reading accuracy, an equation developed by Panda et al. [18] was used to compensate for the temperature and humidity effects on the speed of sound in air, as can be seen in equation (1):

$$
\begin{aligned}
C_{s}= & (331.296+[0.606 \times \theta]) \times(1+[R H \times 9.604 \\
& \left.\left.\times 10^{-6} \times 10^{0.032 \times\left(\theta-\left(0.004 \times \theta^{2}\right)\right)}\right]\right),
\end{aligned}
$$

where $C_{s}$ is the speed of sound in air after compensation, $\theta$ is the air temperature, and $R H$ is the relative humidity of air.

While using the equation as is resulted in better accuracy, the results were overcompensated, and the equation parameters need to be calibrated to our own hardware setup. To do that, a series of tests were conducted to acquire several thousands of reading for distances from 0.5 to 4 meters, under different temperature and humidity conditions, using multiple hardware setups to account for small variations between individual components. The results of these experiments were analyzed, and the equation parameters were optimized accordingly using the GRG nonlinear optimizing method. The results of the parameter optimization process is shown in equation (2):

$$
\begin{aligned}
C_{s}= & (331.296+[0.43657 \times \theta]) \times(1+[R H \times 24.5456 \\
& \left.\left.\times 10^{-6} \times 10^{0.06137 \times\left(\theta-\left(0.01632 \times \theta^{2}\right)\right)}\right]\right) .
\end{aligned}
$$

The optimized equation resulted in substantially greater accuracy and much smaller measurement variations with temperature and humidity change. The results of this optimization process is demonstrated in Figure 6, where the three cases were plotted against each other, noting that the actual distance to be measured was $2078 \mathrm{~mm}( \pm 2 \mathrm{~mm}$, due to the irregular shape of the sensor's tip). It is clear that that the optimized equation produced much more consistent results and have substantially mitigated the effects of the temperature and humidity variations. The microfluctuations are due to the sensor's measurements accuracy, and those cannot be overcome.

(iv) Third Stage. To identify other environmental conditions that may be present in the field and may affect the accuracy and reliability of the measurements, a different approach was taken. Multiple units were installed in remote uncontrolled areas very similar to where they would be installed during actual deployment, while more units were set up in outdoor testing facilities under unfavorable testing conditions. Several different data acquisition systems were installed in these locations to verify some of the measurements and to collect additional data such as atmospheric pressure and wind speed. The results of these tests confirmed that the uncontrolled environmental conditions could cause unexpected issues in the measurements. These particular measurements were characterized by either abnormally very low or very high readings. This is very problematic, as typical averaging techniques of the readings will only get things worse, since it will not be possible to identify these outliers afterwards. By analyzing the data collected by the different test setups, it was found that wind, vibrations, and uneven measurement surfaces were the main contributors to these outliers.

To solve this problem, a statistical filtering approach was followed. After taking several distance readings, the code will first filter any reading that is out of the operational range of the device. After that, it will neglect a range of the highest and lowest readings depending on the number of the original valid 


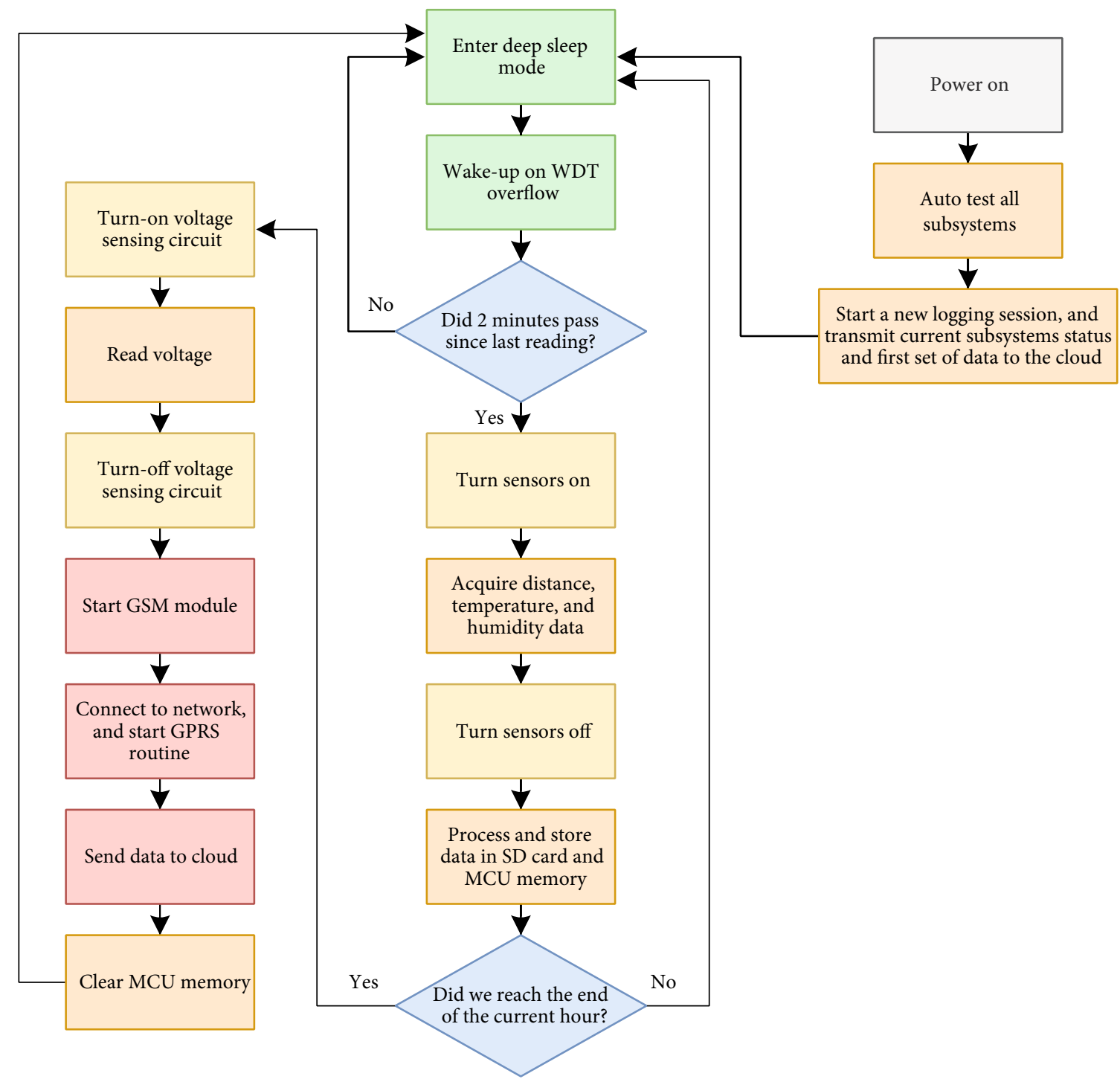

FIGURE 8: Block diagram of the tipping bucket monitoring device workflow.

readings. The remaining values will be averaged to produce the final measurement value. However, these values cannot be arbitrarily chosen. The number of readings to be taken will substantially affect the lifetime of the battery, and the number of readings to be neglected will affect the accuracy of the results. To accurately determine these parameters, a probabilistic model of the outliers was developed. The system was tested under different wind and vibrations levels, taking measurements for a range of distances and doing that on different types of surfaces. Using the results of those tests in addition to the previous field collected data, the filter parameters were optimized.

The final produced filter was undemanding enough to be implemented in the system's microcontroller without requiring overly high processing power or extended on-time periods, while providing very good results, as can be seen in Figure 7. The figure demonstrates the effectiveness of this filtering method by comparing the results taken by a simple averaging technique, compared to the developed filter. The measurements demonstrated were taken in an in-field water channel susceptible to heavy wind gusts, and having a very uneven surface. The average distance measured using the unfiltered readings is $1562.3 \mathrm{~mm}$, compared to an average of $2981.5 \mathrm{~mm}$ for the filtered readings. Considering that the actual distance to be measured was $2978 \mathrm{~mm} \mathrm{(} \pm 5 \mathrm{~mm}$ due to the uneven measured surface), the filtered results are substantially better.

(v) Additional Tests. To further analyze the effects of temperature on the system, the system was put through multiple cycles of both slow and rapid temperature change, from zero to 60 Celsius, but no noticeable effects on data or system stability were found. In addition, another set of tests involving the sensor alone was made; this involved putting 


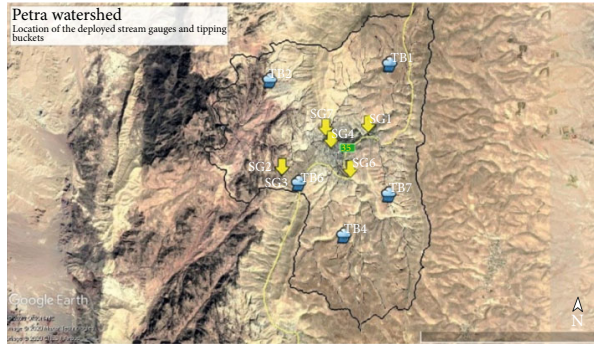

(a)

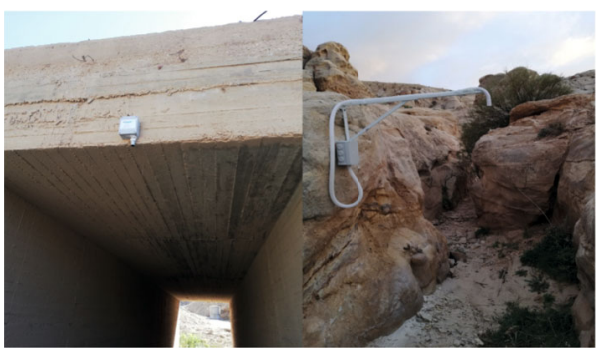

(c)

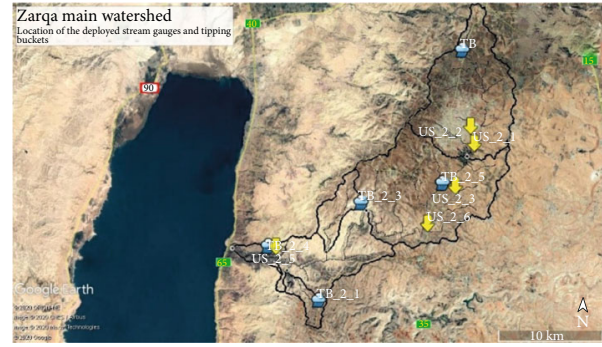

Legend

\Stream

Tipping bucket

(b)

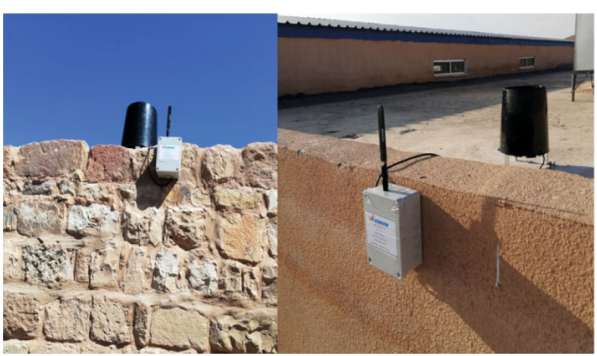

(d)

Figure 9: Deployment locations for stream stage and tipping bucket monitoring devices in (a) Petra watershed and (b) Zarqa Main watershed. Sample pictures of deployed (c) stream stage and (d) tipping bucket monitoring units.

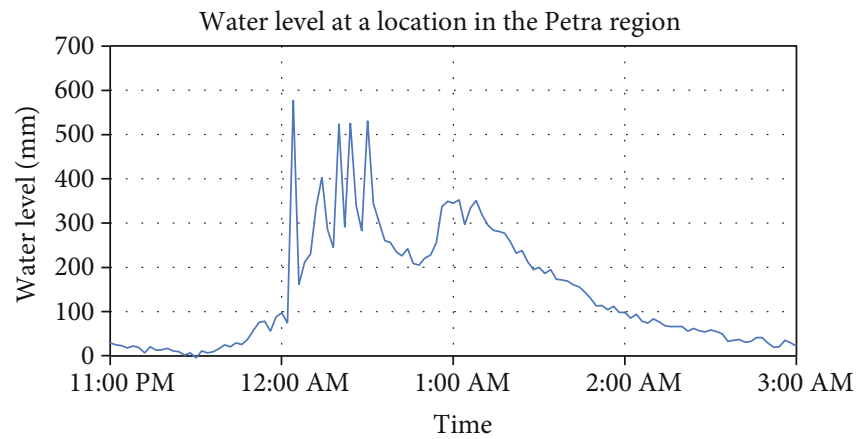

FIGURE 10: Capturing of a fast water increase event.

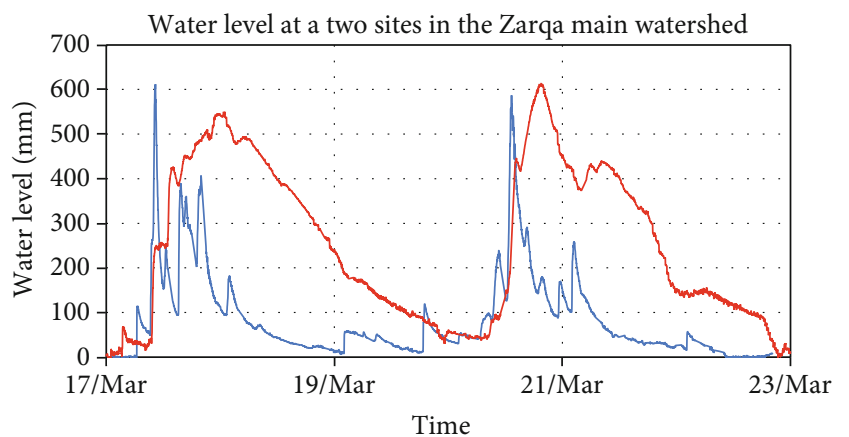

- Site 1

- Site 2

Figure 11: Data acquired from the same hydrological region showing substantial differences in the captured profile. the transducer under different thermal conditions, from minus 10 degrees to 70 degrees Celsius, but no considerable effect was noticed.

2.4. Tipping Bucket Precipitation Measurement. Precipitation rate is one of the most essential meteorological data types for a successful hydrological flood monitoring and modeling effort. However, most currently available meteorological data in the region are averaged across a relatively long period of several hours or more. As several flood events are caused by thunderstorms, and very rapid weather changes, these important details are ultimately lost in the averaging process. Moreover, these metrological events are usually localized and show very high spatial variations, making it essential to have a lot of acquisition points scattered spatially. Bearing that in mind, having a precipitation rate monitoring device that is 

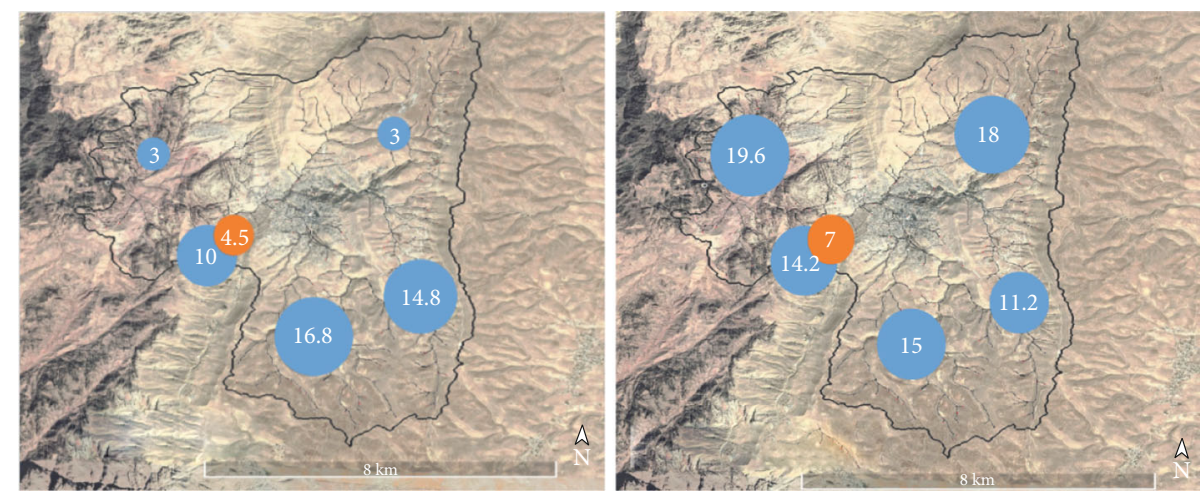

- Total daily precipitation ( $\mathrm{mm}$ )

- Official daily record $(\mathrm{mm})$

FIGURE 12: Spatial variability in recorded total daily precipitation after two arbitrary storms.

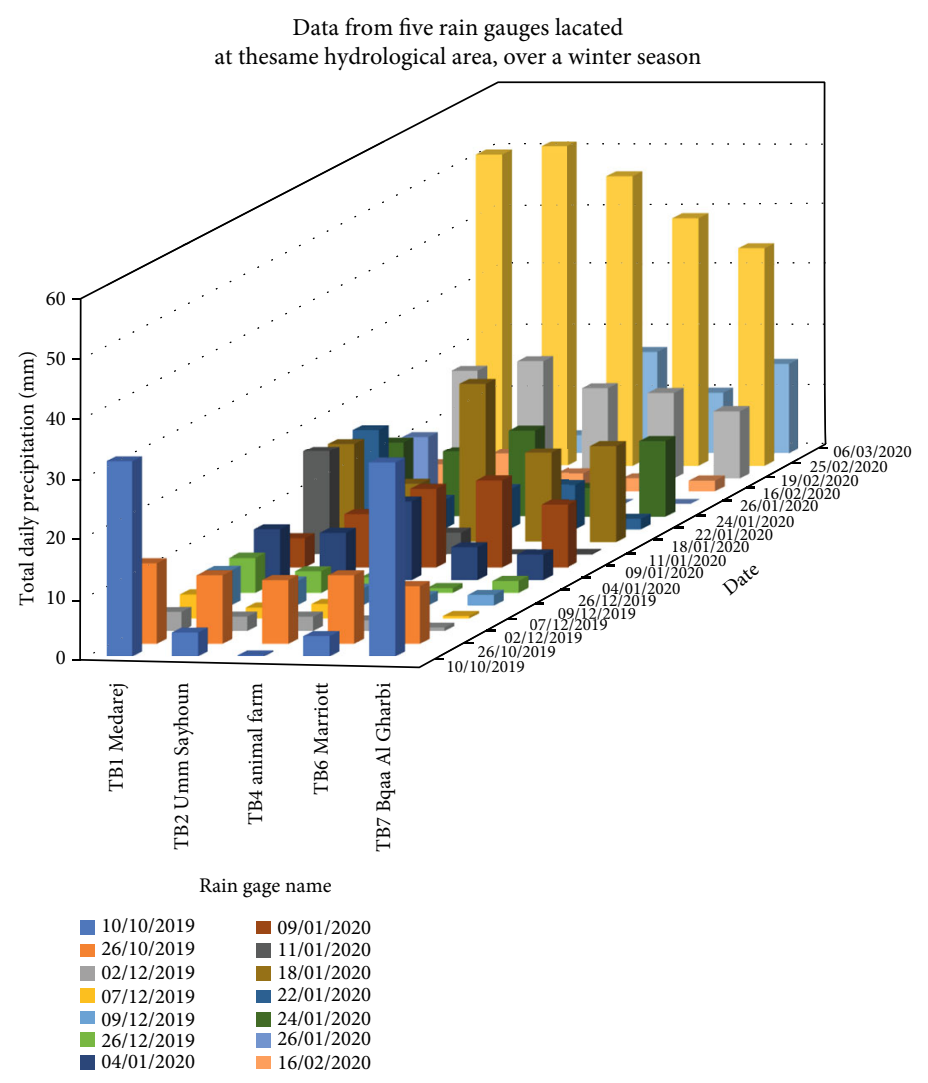

FIGURE 13: Spatial variability in recorded rainfall data over a winter season.

capable of registering these fast changes, while being practical to mass deploy, is the key. Due to the modular design of the HydroMon3 platform, it can perform as a precipitation meter and transmitter by simply connecting a rainfall sensor and performing some software modifications.

A tipping bucket sensor is the most common type of devices used to register precipitation rate. It allows for accurate measurements, while having a very simple mechanism, making it a very reliable sensor for long time remote measurements. The sensor used in this work is the Rika RK400-04 tipping bucket rainfall sensor. The working principle of this sensor depends on the small "buckets" filling up with a calibrated volume of water before "tipping" to spill over, causing a signal to be sent. Since the volume of the bucket is known, and the area of the sensor's funnel is also known, then each signal sent will represent a certain amount of rainfall. Additionally, if the signals were timed, then it could also give information about the rainfall rate, which is the target of this work.

While this system is based on the same platform software as the flood level monitoring device, it differs from it in that the MCU does not need to initiate the measurement process, rather the sensor itself will produce the signal. This will 
generally allow for a more passive working principle and thus will generally have a substantially longer operating times, and subsequently battery lifetime. The general working principle of the rainfall sensor is shown in Figure 8.

The system starts up with the same diagnostic routine, before it starts the main loop. The tipping bucket sensor is connected to a physical interrupt pin on the MCU, where every time the sensor "tips," the MCU registers one tip through the interrupt routine, regardless of the current state of the MCU. This allows the MCU to keep track of the sensor's operation with minimal wake-up time. The system will save all the data collected to the SD card, but will only transmit data if it registers a rainfall event, or as a heartbeat signal at the end of the day.

At normal rainfall rates, the device will transmit data once per hour, but at higher rates, that could indicate a storm or a thunderstorm event; the device will transmit data every 10 minutes, allowing for a more real-time data acquisition. Due to the passive nature of the workflow and the lower transmission rate most of the time, the energy requirements of the more intense transmission pattern during a storm can be justified. The data acquisitions resolution and transmission frequency can be modified in the software to adapt for different data and energy requirements.

Since the output of the tipping bucket is a low-speed digital signal, no filtering or calibration is needed. The device will store and return data as "number of tips" without calculating the amount of rainfall, where this postprocessing is done at the receiving end. This is done so the device can be flexibly connected to different-capacity tipping bucket sensors without the need to adjust the software parameters.

\section{Results and Discussion}

The results presented in this section are a showcase of the actual deployment of 20 devices in two flood-active hydrological regions in Jordan, Dead Sea, and Petra.

3.1. Data Acquisition Performance. Figure 9(a) shows the location of the installation sites in Petra, while Figure 9(b) shows those in the Zarqa Main Watershed. It should be noted that the locations were chosen strategically to cover the majority of the area's spatial extents while taking topography in consideration. Each subbasin was equipped with a rain gauge and a stream gauge to monitor the rainfall-runoff processes. Mass deployment of the multiple HydroMon3 (sample deployed devices can be seen in Figures 9(c) and 9(d)) and sensor arrays allowed for real-time monitoring of precipitation rate and magnitude as well as the corresponding runoff hydrograph; both data types are accompanied with a time signature allowing for a cause-effect evaluation.

Measurements acquired from the deployed devices presented here demonstrate the capabilities and the effectiveness of the HydroMon3 platform. Figure 10 shows the stream stage device capturing a high-resolution flow event due to the high data acquisition rate. Although the entire event lasted about two hours, the reported data shows a detailed profile of the event, with a relatively high accuracy.

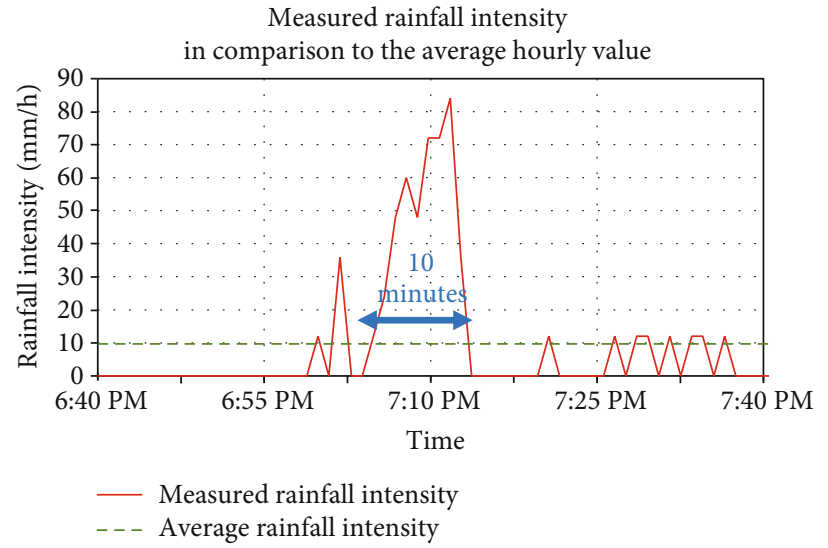

FIgURe 14: Demonstrating the high data resolution acquired by the tipping bucket monitoring device.

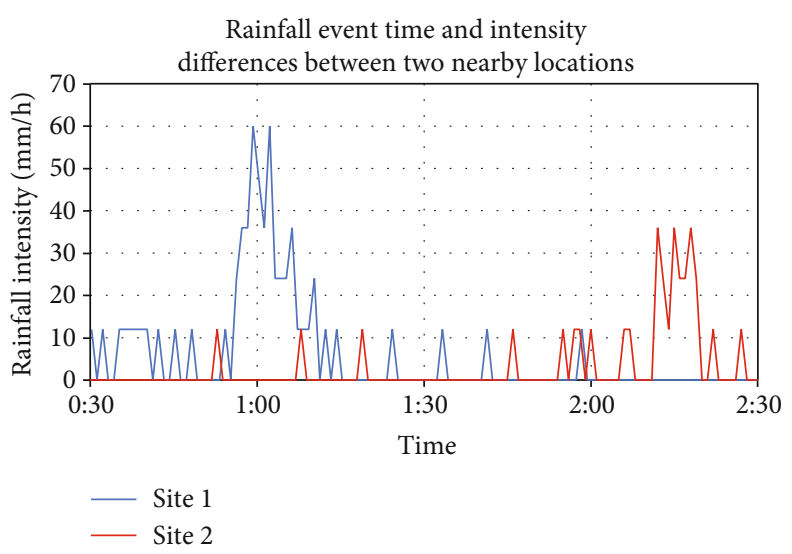

FIgURe 15: Difference in the time and intensity of a storm in two sites located at the same hydrological region.

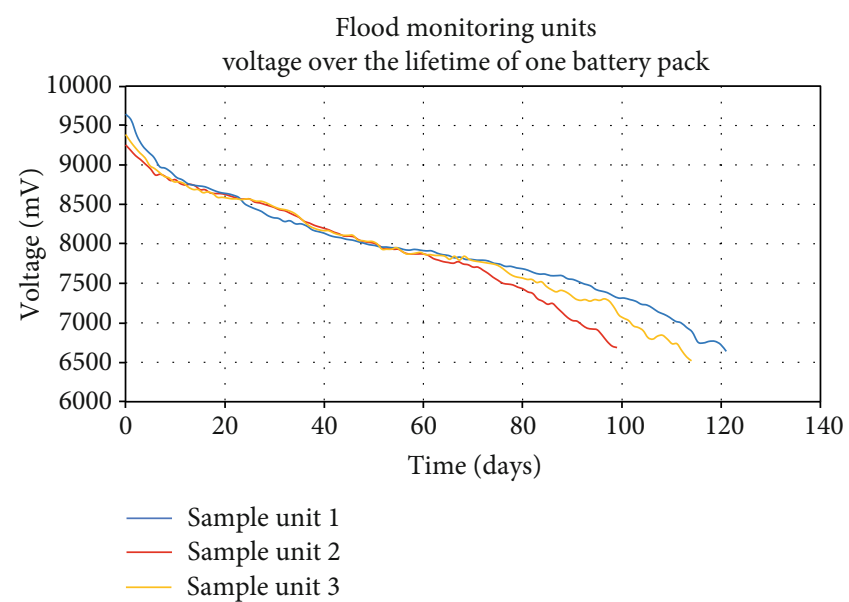

FIgURE 16: Voltage profile and lifetime of the flood monitoring device's battery pack.

In hydrology, spatial variations can be very high. Therefore, and in order to properly capture the variation, it is advantageous to deploy numerous low-cost devices. To illustrate the 
TABLe 2: Verification of the initial measurements with field lifetime.

\begin{tabular}{|c|c|c|c|c|c|}
\hline Component & $\begin{array}{l}\text { Average } \\
\text { current } \\
\text { (A) }\end{array}$ & $\begin{array}{l}\text { Duration of an } \\
\text { operations cycle } \\
\text { (seconds) }\end{array}$ & $\begin{array}{c}\text { Energy } \\
\text { consumption/cycle of } \\
\text { operation }(\mathrm{mWh})\end{array}$ & $\begin{array}{l}\text { Number of } \\
\text { cycles/hour }\end{array}$ & $\begin{array}{c}\text { Energy } \\
\text { consumption/110 } \\
\text { days }(\mathrm{mWh})\end{array}$ \\
\hline GSM & 35.16 & 38 & 2.923 & 1 & 7,718 \\
\hline Ultrasonic sensor & 20 & 3.3 & 0.145 & 30 & 11470.8 \\
\hline SD card module & 17.4 & 2.44 & 0.093 & 1 & 245.9 \\
\hline $\mathrm{MCU}+$ passive components & 0.03 & 3,600 & 0.237 & 1 & 625.7 \\
\hline $\begin{array}{l}\text { Total energy consumption/110 days } \\
\text { (mWh) }\end{array}$ & \multicolumn{5}{|c|}{$20,060.4 \mathrm{mWh}$} \\
\hline $\begin{array}{l}\text { Estimated usable capacity of six } \\
\text { Energizer } \mathrm{Max}^{\circledR} \text { alkaline AA batteries }\end{array}$ & \multicolumn{5}{|c|}{$19,750-21,725 \mathrm{mWh}$} \\
\hline
\end{tabular}

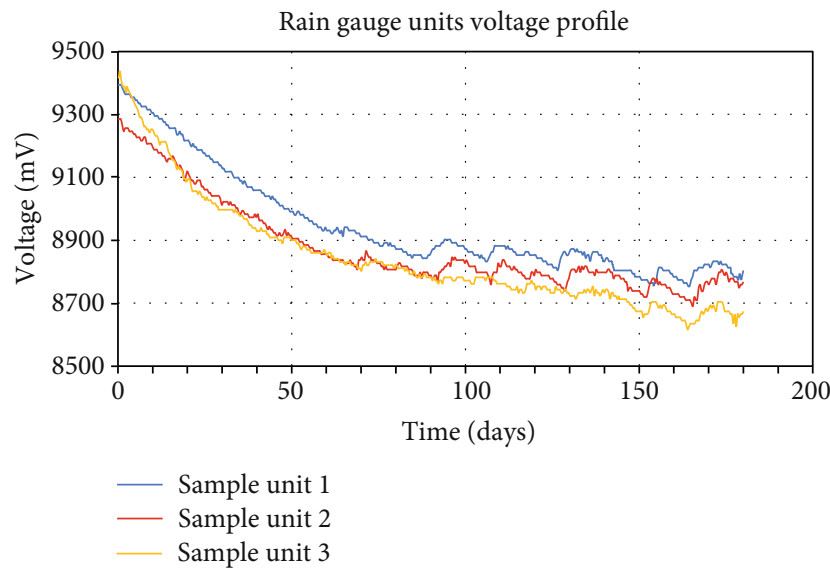

FIGURE 17: Voltage profile of three sample units of the rain gauges, showing only slight drop in a period of 6 months.

concept, Figure 11 shows two subsequent events occurring at two different locations (6 kilometers apart) at the same hydrological region. These events were accurately captured with the exact time relative to each other, showing the substantial difference in the profile of each location; this can be attributed to the shape of the subbasin, topography, and retention characteristics among other possible factors. Moreover, the subsequent event confirms the accuracy and the repeatability of the device's results.

Data acquired by the several deployed rain gauge devices over more than a year of stable operations and consistent measurements confirm reliable and accurate data acquisition and demonstrate the benefits of the high temporal data resolution, and the wide spatial coverage of the hydrological region. Similar to what was illustrated in Figure 11, deployment of several devices was instrumental in highlighting the spatial variability of precipitation quantities. Figure 12 shows recorded total daily precipitation amounts $(\mathrm{mm})$ at the HydroMon3 device locations as well as the reported data from the official meteorological department (shown in orange color) for two sample dates. The figure clearly highlights the necessity of intense deployment of these devices to properly capture the rainfall-runoff patterns in such small-scale settings.

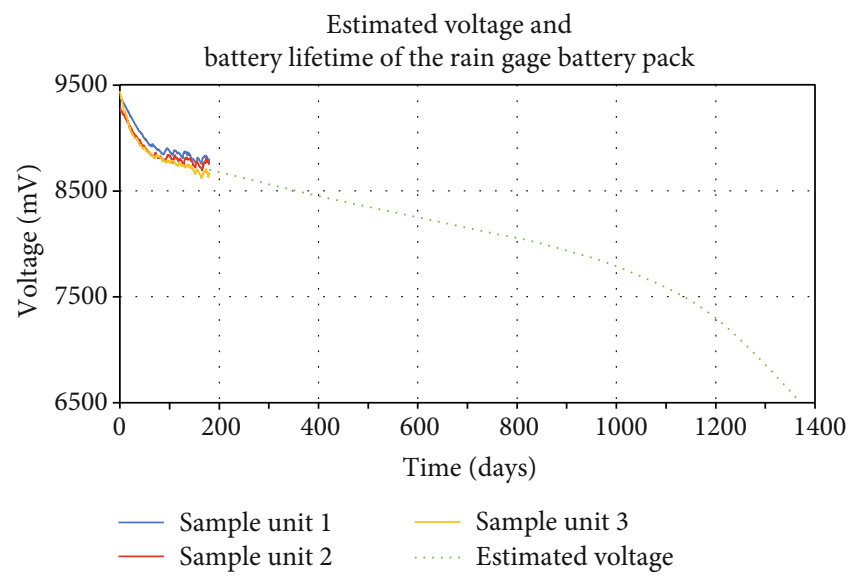

FIGURE 18: Forecasting the discharge curve of the rainfall meter battery pack.

Figure 13 further demonstrates the variation in readings, both spatially and temporally. The figure summarizes the total daily precipitation recorded at 5 stations distributed throughout the watershed of interest for 17 storm events throughout the 2019-2020 rainy season. Significant variations are noted in some of these events (e.g., the 10-10-2019 storm) which clearly demonstrates the need for several monitoring stations in order to properly capture the precipitation pattern and accurately study and model the system's performance. It is worth reemphasizing that the intents of this paper are to demonstrate the monitoring system and not discuss the resulting data; that will be done in a separate effort.

Rainfall intensity is equally important for hydrological studies involving flash flood analysis. Figure 14 shows the detailed profile of a thunderstorm event occurring over a period of about 10 minutes; rainfall intensity is presented in $\mathrm{mm} / \mathrm{hr}$. Such events are the main drivers to flash floods. It can be clearly seen that a reported record of hourly or daily frequency will miss these short bursts of precipitation. Thus, if modeling runoff due to thunderstorm events is required, then these low-resolution records will not be sufficient, hence the need for our customizable, highly deployable systems.

Figure 15 demonstrates rainfall intensity at two different sites in the same hydrological region, showing the difference 
TABLE 3: Verification of the compatibility of the initial energy measurements for the rain gauge device field test results.

\begin{tabular}{lccccc}
\hline Component & $\begin{array}{c}\text { Average } \\
\text { current (A) }\end{array}$ & $\begin{array}{c}\text { Duration of an } \\
\text { operations cycle } \\
\text { (seconds) }\end{array}$ & $\begin{array}{c}\text { Energy consumption/cycle } \\
\text { of operation (mWh) }\end{array}$ & $\begin{array}{c}\text { Number of } \\
\text { cycles/hour }\end{array}$ & $\begin{array}{c}\text { Energy } \\
\text { consumption/110 days } \\
(\mathrm{mWh})\end{array}$ \\
\hline $\begin{array}{l}\text { GSM } \\
\text { SD card module }\end{array}$ & 35.16 & 38 & 2.923 & 0.0799 & 1 \\
$\begin{array}{l}\text { MCU + passive } \\
\text { components }\end{array}$ & 17.4 & 2.44 & 0.093 & 1 & 616.7 \\
$\begin{array}{l}\text { Total energy } \\
\text { consumption/110 days } \\
(\mathrm{mWh})\end{array}$ & 0.03 & 3,600 & 0.237 & & 245.9 \\
\hline
\end{tabular}

in the time the storm reached each site, and the difference in the profile and intensity.

3.2. Energy Performance. This factor of the system performance is extremely important. These devices are typically installed in remote locations with limited accessibility. The need for a very small footprint is high in order to minimize vandalism. Thus, one of the success criteria that we set for the developed devices is the extremely low-energy consumption and relatively cheap energy source.

Most deployed devices of the flood monitoring system were supplied with six Energizer Max $^{\circledR}$ alkaline AA batteries in series to standardize conditions for evaluation purposes. Of-the-shelf batteries were preferred to continue the general theme of a low-cost and easy-to-reproduce device. However, different battery technologies and types within the safe operating voltage can be directly plugged into the platform. Different workflows, sensors, acquisition frequencies, and transmission frequencies are what determines the overall energy consumption. This is clearly shown in the difference in energy consumption between the two devices.

In the flood level monitoring devices, the diagnostic data acquired by the deployed units show that the device held reliable operation until a pack voltage of 6.5 to $7 \mathrm{~V}$ was reached. Accordingly, this resulted in a lifetime of 100 to 120 days of operation. The voltage profiles of three sample devices are shown in Figure 16. By interpolating the voltage readings and the battery's datasheet figures, the average current consumption of the stream stage monitoring device is 950 $\mu \mathrm{A}$, while the average power consumption is $7.39 \mathrm{~mW}$.

To verify the soundness of our initial measurements and estimations of the system's energy consumption, a breakdown of the components energy consumption and estimated operation time are shown in Table 2 for a 110-day interval. The estimated consumption is compared to the expected usable capacity of six Energizer Max $^{\circledR}$ alkaline AA batteries according to their datasheet. The table shows how the expected energy consumption of the system falls within the expected capacity of the batteries, proving the validity of our initial work. Depending on the GSM signal strength, distance to be measured, temperature, and small variation in the electronics components, the lifetime of the batteries will vary, as was seen in the three cases demonstrated in Figure 16.

The tipping bucket rain gauge, on the other hand, due to its passive operating nature and the digital nature of the acquired data, only transmits - for the most part-when an actual event of interest occurs. Bearing in mind that the transmission routine is the most energy-demanding operation, this substantially reduces the active energy consumption, which translates into a relatively very long battery lifetime. Figure 17 shows the voltage profile of three sample devices over a period of six months.

As clearly seen in Figure 17, the voltage level is far from reaching the end-of-life voltage level. To approximate the expected lifetime, the data from the flood monitoring device performance and the datasheet performance curves were used to forecast the curve shape as can be seen in Figure 18. The figure shows that one battery pack should theoretically last more than 3.5 years. Building upon lab measurements and deployed unit data, the average current consumption of the rain gauge meter device is $82 \mu \mathrm{A}$, and its average power is $638 \mu \mathrm{W}$. Due to the variable frequency of acquisition and transmission in this device, energy consumption will increase with the increase of rainfall frequency, making the battery lifetime climate-dependent.

As in the case of the flood level monitoring devices, the initial energy consumption measurements of the components validate those findings, as the GSM module only operated around 700 times during that year, and the tipping bucket sensor is passively operated and thus requires no power. Table 3 shows how low the energy consumption is in this device, noting that the batteries are more likely to self-discharge before they are used up by the device. The results demonstrated in the table are for 110-day interval for easier comparison with the flood level monitoring device.

For testing purposes, a few units were equipped with $\mathrm{NiMH}$ and lithium AA batteries. NiMH was found to provide little added value for the much higher cost, plus being less favorable for very low power applications due to their generally higher self-discharge rate. Lithium AA batteries, on the other hand, showed better performance and more stable voltage, but the higher price and lower availability make them less desirable to standardize.

\section{Conclusion}

The significance of this work manifests in the ability of utilizing the mass production of IoT-related modules and electronic parts to minimize costs and maximize flexibility, while achieving reliable performance and data acquisition capabilities that rivals other much more expensive commercial solutions. The average cost of the main platform was about 50 USD, while 
the ready to deploy unit had an average cost of 90 to 150 USD depending on the type and number of sensors used; this is far below $10 \%$ of commercially available solutions, and it provides much higher flexibility. The quality of gathered data and the device's low energy requirements were empirically evaluated and verified by the data gathered in lab a prototype testing, as well as from over 20 field-deployed units.

Comparison of local data showed the significance of spatial distribution and temporal resolution on the variability of acquired data and stresses the need to cover these aspects, where the success of this project opens the door to wide-scale coverage of key hydrological locations to achieve the needed spatial and temporal data resolution.

For future work, a soil moisture monitoring device based on the HydroMon3 platform is currently being developed to further enhance the capability of full hydrological monitoring. The energy performance of the stream stage monitoring device was adequate for one season at current settings, but it can be further enhanced by optimizing the transmission routine to only transmit when an event of interest occurs. Current data from the field units would aid in developing these algorithms.

\section{Data Availability}

Data available on request. Contact Ahmad Al-Hmoud from the German Jordanian University (ahmad.alhmoud@gju.edu.do).

\section{Conflicts of Interest}

The authors declare that there is no conflict of interest regarding the publication of this paper.

\section{Acknowledgments}

This research was partly funded through the U.S. Ambassadors Fund for Cultural Preservation (AFCP 2017) and was partly funded through a grant from the Deanship of Scientific Research at the German Jordanian University (grant number: SNRE 01/2018). The authors would like to thank Dr. Zakariya Dalala and Dr. Osama Saadeh for supporting this research by providing access to their laboratory. We would also like to acknowledge the staff at the Petra Development Board and the different facilities and entities that hosted the devices and all the engineers who worked tirelessly on developing, debugging, and commissioning the devices.

\section{References}

[1] R. Marin-Perez, J. García-Pintado, and A. S. Gómez, "A realtime measurement system for long-life flood monitoring and warning applications," Sensors, vol. 12, no. 4, pp. 4213-4236, 2012.

[2] G. Guimares Nobre, B. Jongman, J. Aerts, and P. J. Ward, “The role of climate variability in extreme floods in Europe," Environmental Research Letters, vol. 12, no. 8, 2017.

[3] K. A. Al-Qudah, "Floods as water resource and as a hazard in arid regions: a case study in Southern Jordan," Jordan Journal of Civil Engineering, vol. 159, 2011.
[4] R. A. Al-Weshah and F. El-Khoury, "Flood analysis and mitigation for Petra area in Jordan," Journal of Water Resources Planning and Management, vol. 125, no. 3, pp. 170-177, 1999.

[5] R. Pon, M. A. Batalin, V. Chen et al., "Coordinated static and mobile sensing for environmental monitoring," in International Conference on Distributed Computing in Sensor Systems, pp. 403-405, Marina del Rey, CA, USA, 2005.

[6] R. Cardell-Oliver, M. Kranz, K. Smettem, and K. Mayer, “A reactive soil moisture sensor network: design and field evaluation," International journal of distributed sensor networks, vol. 1, no. 2, pp. 149-162, 2005.

[7] A. Atijosan, A. O. Salau, R. Ariyo Badru, and T. Alaga, "Development of a low cost community based real time flood monitoring and early warning system," International Journal of Scientific Research in Science, Engineering and Technology, vol. 3, pp. 189-195, 2017.

[8] S. I. Abdullahi, M. H. Habaebi, and N. A. Malik, "Flood disaster warning system on the go," in Proceedings of the Proceedings of the 2018 7th International Conference on Computer and Communication Engineering, ICCCE 2018, pp. 258-263, Kuala Lumpur, Malaysia, 2018.

[9] X. Yang, K. Ong, W. Dreschel, K. Zeng, C. Mungle, and C. Grimes, "Design of a wireless sensor network for long-term, in-situ monitoring of an aqueous environment," Sensors, vol. 2, no. 11, pp. 455-472, 2002.

[10] L. Ruiz-Garcia, L. Lunadei, P. Barreiro, and J. I. Robla, “A review of wireless sensor technologies and applications in agriculture and food industry: state of the art and current trends," Sensors, vol. 9, no. 6, pp. 4728-4750, 2009.

[11] M. Bartos, B. Wong, and B. Kerkez, "Open storm: a complete framework for sensing and control of urban watersheds," Environmental Science: Water Research \& Technology, vol. 4, no. 3, pp. 346-358, 2018.

[12] B. K. Nuhu, O. T. Arulogun, I. A. Adeyanju, and I. M. Abdullahi, "Wireless sensor network for real-time flood monitoring based on 6loWPAN communication standard," APTIKOM Journal on Computer Science and Information Technologies, vol. 1, pp. 12-22, 2016.

[13] A. Kruger, W. F. Krajewski, J. J. Niemeier, D. L. Ceynar, and R. Goska, "Bridge-mounted river stage sensors (BMRSS)," IEEE Access, vol. 4, pp. 8948-8966, 2016.

[14] S. W. Lo, J. H. Wu, F. P. Lin, and C. H. Hsu, "Cyber surveillance for flood disasters," Sensors, vol. 15, no. 2, pp. 23692387, 2015.

[15] S.-W. Lo, J.-H. Wu, F.-P. Lin, and C.-H. Hsu, "Visual sensing for urban flood monitoring," Sensors, vol. 15, no. 8, pp. 20006-20029, 2015.

[16] C. Moreno, R. Aquino, J. Ibarreche et al., "RiverCore: IoT device for river water level monitoring over cellular communications," Sensors, vol. 19, no. 1, p. 127, 2019.

[17] O. Saadeh, A. Al-Hmoud, and Z. Dalala, "Characterization circuit, gate driver and fixture for wide-bandgap power semiconductor device testing," Electronics, vol. 9, no. 5, p. 703, 2020.

[18] K. G. Panda, D. Agrawal, A. Nshimiyimana, and A. Hossain, "Effects of environment on accuracy of ultrasonic sensor operates in millimetre range," Perspectives on Science, vol. 8, pp. 574-576, 2016. 